\title{
Transition Period for ESO
}

All the main components of the $3.6 \mathrm{~m}$ optical telescope of the European Southern Observatory have now been shipped from Europe to Chile. The telescope building including the dome is virtually complete and dominates the Observatory site on the top of the mountain of La Silla, situated on the edge of the Atacama desert, $600 \mathrm{~km}$ north of Santiago. Assembly and testing are expected to take about six months and the first observations are due to be made in November or December. As a result ESO is moving into a new era in its history as the accent shifts from construction to operation and La Silla assumes a place amongst the great Observatories of the world. This is not to underrate the importance of the work that has been going on at the Observatory since 1966 using a range of smaller telescopes. But it is from this year that the Organization will really be able to make its mark on astronomy. The $1 \mathrm{~m} \mathrm{Schmidt} \mathrm{is} \mathrm{now}$ systematically producing high quality star maps of the southern skies in the blue and providing the basic catalogue for the high resolution observations with the $3.6 \mathrm{~m}$ when it comes into action.

In anticipation of this new maturity the Council of ESO took important steps at its last meeting to give a more streamlined shape to the Organization in the coming years. ESO has been a somewhat diffuse organization with several establishments in Chile and two main groups in Europe, - the headquarters group in Hamburg (FRG) and the Telescope Project Division and Sky Atlas Laboratory at CERN in Geneva.

Already consolidation moves have been made in Chile and the majority of staff have moved to the Observatory leaving only a small contingent in Santiago. Now the Council has accepted an offer made by the government of the Federal Republic of Germany to provide a site and buildings for the Organization at Garching, $15 \mathrm{~km}$ north of Munich close to the Max Planck Institute of Plasma Physics. The programme of ESO is for the architect engineer to be selected in May, for construction to begin in April next year and a general move of all European operations into the new centre early in 1979. The office in Hamburg will, however, move on
1 July this year into temporary accommodation near the new site.

This difficult decision now out of the way, the Director-General of the Organization, Prof. L. Woltjer can turn his attention to finding the optimum balance between the use of the facilities by in-house and visiting astronomers and the ideal equilibrium between observation, development and administration within the budgets established by the Member States. Amongst these members (Belgium, Denmark, France, German Fed. Rep., Netherlands and Sweden) the two big partners have major projects of their own in hand which are inevitably in some competition with the international body at least for funding. Both France and Germany are building $3.6 \mathrm{~m}$ telescopes - France (with Canada and Hawaii) in Hawaii, and Germany in Spain.

ESO was somewhat slow in getting down to the completion of its major projects and as a result there has not been quite the whole-hearted commitment to the international body as is evident at CERN. ESO would undoubtedly be strengthened by the addition of further members and is not shy in acknowledging this but now is hardly the time in Western Europe when governments are looking for new candidates for research funds.

The successful conclusion of the tests on the $3.6 \mathrm{~m}$ at the works of Creusot-Loire in France gives confidence that the telescope will fulfill its designer's hopes and the unique seeing qualities of the La Silla site coupled to its ideal location at latitude $30^{\circ} \mathrm{S}$ should ensure that ESO has a unique instrument in its hands.

Of essentially " conventional " design incorporating a horseshoe on a polar mounting, its final performance owes much to the ingenuity of the ESO team and the adaptability of the computer control system. Two major problems presented themselves when testing first started. The friction in the polar drive was much higher at certain displacements than was acceptable and the whole assembly had a tendancy to oscillate after displacement with a frequency of about $0.6 \mathrm{~Hz}$, the damping being disconcertingly small. The solution to the first was to profile the horseshoe bearing surface to a multi-order curve to maintain a constant bearing area with the support pads over the whole of the arc. The solution to the second was to feed into the control system, information on structural accelerations so that the main drive motors (working through sput gears) can by dynamic feed-back damp out the oscillations. The computer control system had already been proved out on smaller instruments at La Silla and shown itself to be a major step forward in telescope guidance techniques. Its ability to cure the vibration troubles in this way indicates that designers of big telescopes will in future no longer be restricted to the traditional configurations of the past.

ESO conceives its role over the next few years, as having a number of facets. Primary requirement is to operate the La Silla observatory efficiently and make maximum use of the instruments available there. It is clear that the distance of the Observatory from Europe imposes its own discipline and the observational programme must be more highly organized than is perhaps normal in the traditionally personalized field of observational astronomy.

It is expected that about $2 / 3$ of the time on the telescopes will be taken by visiting astronomers and about $1 / 3$ by astronomers appointed to the staff of ESO for a period of a few years, or in a limited number of cases for longer. It must be expected also that the practice of working in teams grouping visitors and staff members will also grow and that some measurements will have to be made at second hand. Pressure on the facilities is going to be very great and already ESO has three times the demand for time than it can cope with.

This places a heavy responsibility on the Observing Programmes Committee which has the job of optimizing the programme from the scientific point of view. Wisely there is no direct link between governments and observing programmes and experience has shown that competition between individuals, with the Organization exercising a benevolent role to ensure fair play, works best.

Parallel to the observational and theoretical work is the important area of instrument development and ESO intends to maintain an active group 
engaged in this work so that it can on the one hand make direct contributions to the technology and on the other act as a natural coordinating centre for the diverse projects going on in Universities and in national institutes. There is a great need for more advanced detectors and the application of image tubes which because of their improved sensitivity over the photographic plate allow direct image studies and an extension of seeing power.

The opening up of the southern skies is revealing a tremendously rich field of study which until very recently has been almost totally ignored in spite of the fact that the centre of our own galaxy is seen best from the South and the Magellamic clouds, only visible from the South, are ten times closer than the nearest stellar system (or galaxy) seen from the north (the Andromeda nebula).

There is as a result a huge quantity of work to do but ESO is not looking inwards. Previous articles have described the projects of ESA in the astronomical field and co-operation between ESA and ESO is now beginning to grow. The study of distant objects is best made at a variety of frequencies and some of the most interesting recent results have come from a combination of observations in the $\mathrm{X}$-ray and $\gamma$-ray regions with optical observations. Contact has also been made with the European Science Foundation which is anxious to help in coordinating European research projects.

Co-operation with the USSR is also beginning. There have always been close contacts with the non-member European States and also with the USA which has an observatory at Cerro Tololo some $75 \mathrm{~km}$ from La Silla. But in a short time the first Soviet astronomer will come and work in the Sky Atlas Laboratory and it is hoped that such informal exchanges of people will lead to a widening spread of contacts between astronomers in Western and Eastern Europe.

The problem facing ESO in the coming years is evidently not one of paucity of opportunity so much as choice of priorities. The first essential is to prove that the $3.6 \mathrm{~m}$ at La Silla is as good as it is believed it will be and then to prove that it is possible to run efficiently an observatory $9000 \mathrm{~km}$ away. Once these are established there will be time to think about future major projects. Astronomy and cosmology move at such a pace, it is difficult to forecast what will be the centre of interest in 10 years time.

\section{INSTITUTE FOR NUCLEAR PHYSICS RESEARCH AMSTERDAM, THE NETHERLANDS}

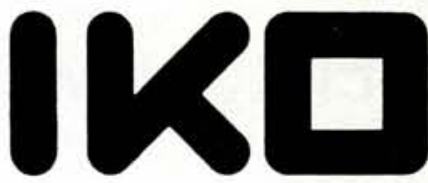

Applications are invited for the tenured position of experimental

\section{SENIOR RESEARCH PHYSICIST}

and possibly project leader in the field of pion/muon physics.

IKO is the intermediate energy physics section of the new Dutch national institute (NIKHEF) for nuclear and high energy physics. At the institute a $500 \mathrm{MeV}$ high duty cycle linear electron accelerator (including a pion/muon facility) is presently under construction. Approximately 200 people work at IKO, of which about 50 are scientists. Apart from external collaborations, the pion/muon group will consist of about $10 \mathrm{IKO}$ physicists.

Applicants for this position are expected to have substantial experience in the field of intermediate energy physics or in a closely related area. They should be well acquainted with the latest theoretical as well as instrumental developments in the field.

Further information can be obtained from Dr. P.F.A. Goudsmit at the Institute (tel. 020-9309 51).

Applications, including curriculum vitae, possible references and a list of publications should be sent to the general scientific director of IKO, Prof. Dr. A.H. Wapstra, P.O. Box 4395, Amsterdam 1006, the Netherlands.

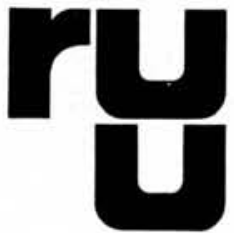

\section{Rijksuniversiteit Utrecht}

In the Physics Department of Utrecht University, The Netherlands, a position will become vacant September, 1976 for a

\section{Full professor of experimental physics}

who will be expected to undertake and initiate research in the field of atomic physics and to participate in physics teaching. $\mathrm{He} / \mathrm{she}$ should have a sound theoretical background. Knowledge of the Dutch language is not a prerequisite.

At present the main interests of the atomic physics group include electron-atom collisions, and surface physics with ion beams.

Further information can be obtained in writing from : Prof. Dr. C. Th. J. Alkemade, Physics Laboratory, Utrecht University, Sorbonnelaan 4, Utrecht, The Netherlands.

Applicants are requested to submit an extensive curriculum vitae, which should provide detailed information on research and teaching experience, and a list of publications etc., together with the names of at least three referees.

Applications should be sent to: Prof. Dr. P.M. Endt, Physics Laboratory, Utrecht University, Sorbonnelaan 4, Utrecht, The Netherlands. 